\title{
BEHNKE-STEIN THEOREM FOR ANALYTIC SPACES
}

\author{
BY
}

\section{ALESSANDRO SILVA}

ABSTRACT. The notion of $q$-Runge pair is extended to reduced complex analytic spaces. A necessary and sufficient condition for a pair of $n$-dimensional analytic spaces to be an $\boldsymbol{n}$-Runge pair is proved and it is shown that this result extends a Behnke-Stein theorem when $n=1$. A topological property of $q$-Runge pairs of spaces is also proved.

Introduction. We extend the notion of $q$-Runge pair introduced for the first time in [12] for complex analytic manifolds to reduced complex analytic spaces. We prove in $\S 1$ that our definition is actually an extension both of the one given in [12] for manifolds and of the classical one of Runge pair of Stein spaces.

We prove in $\S 2$ a result which extends to spaces the classical Behnke-Stein "Rungescherapproximationssatz" for Riemann surfaces, by means of duality theorems.

A topological property of $q$-Runge pairs of analytic spaces is proved in $\S 3$; this last result is not complete, since we do not get information about torsion of homology groups with values in the constant sheaf $\mathbf{C}$. We finally apply the notion of $q$-Runge pair to the problem of computing the degree of cohomological completeness of the limit of an increasing sequence of analytic spaces with given degree of cohomological completeness, as we have done in [9] for manifolds.

0 . Notations and main definition.

(0.1) In the following, we shall always denote by $X$ a noncompact, reduced, countable-at-infinity, complex analytic space with $\operatorname{dim}_{\mathrm{C}} X=n ; Y$ in the following will always be an open analytic subspace of $X$.

(0.2) If $X$ is a "modele" in some open subset $U$ of $\mathbf{C}^{n}$ defined by the ideal sheaf $I$, we can define the sheaf of holomorphic $p$-forms on $X$ by

$$
\Omega_{X}^{p}=\Omega_{U}^{1} / d I \wedge \Omega_{U}^{p-1}+I \Omega_{U}^{p} .
$$

Received by the editors October 28, 1973 and, in revised form, December 18, 1973. AMS (MOS) subject classifications (1970). Primary 32E15; Secondary 32F10.

Key words and phrases. $q$-Runge pairs, cohomologically $q$-complete analytic spaces, Hausdorff cohomology, dualizing complex and derived functors. 
If there is no ambiguity $\Omega_{X}^{p}=\Omega^{p}$. For arbitrary $X, \Omega_{X}^{p}$ is defined by gluing. It is well known that $\Omega_{X}^{p}$ is a coherent sheaf (we denote this property by $\Omega^{p}$ $\in \operatorname{Coh}(X))$.

If $X$ is a manifold, let $\left\{\Gamma\left(X, \Omega^{p, \cdot}\right) ; d_{p,{ }^{\prime \prime}}^{\prime \prime}\right\}$ be the complex induced for cross-sections form Dolbeault's resolution of $\Omega^{p}$.

(0.3) By $H_{c}^{\cdot}(X, F),\left(H^{\cdot}(X, F), H_{c}^{\cdot}(X, F)\right)$, we denote the cohomology groups, with values in the sheaf $F \in \operatorname{Coh}(X)$, with compact support (resp. the Hausdorff cohomology groups, the Hausdorff cohomology groups with compact support). By $\operatorname{Ext}_{c}\left(X ; F, K_{\dot{X}}\right)$, Ext ${ }^{\cdot}\left(X ; F, K_{\dot{X}}\right)$, Ext $_{c}\left(X ; F, K_{\dot{X}}\right)$, with $F \in$ $\operatorname{Coh}(X)$ and $K_{X}$ the dualizing complex introduced in [6], we denote, as above, compactly supported, Hausdorff, and Hausdorff compactly supported Ext $_{c}{ }_{c}$ groups, respectively.

(0.4) The connections between these groups are stated in the following duality result (premier théorème de dualité absolue, Ramis-Ruget [6]): For every $F \in \operatorname{Coh}(X)$ and for every $q \in \mathbf{N}$, a unique structure of the quotient of the Fréchet-Schwartz space on $H^{q}(X, F)$ and a unique structure of the quotient of the dual of the Fréchet-Schwartz space on $\operatorname{Ext}_{c}^{-q}\left(X ; F, K_{X}\right)$ exist such that a natural linear form on $\Gamma_{c}\left(X, K_{X}\right)$ induces a pairing between $H^{q} q(X, F)$ and $\operatorname{Ext}_{c}^{-q}\left(X ; F, K_{\dot{X}}\right)$. Furthermore $H^{q}(X, F)$ is a Hausdorff space if and only if $\operatorname{Ext}_{c}^{1-q}\left(X ; F, K_{\dot{X}}\right)$ is a Hausdorff space.

(0.5) We shall need also the following criterion (Ramis-Ruget-Verdier [7]). Let $F \in \operatorname{Coh}(X)$. $\operatorname{Ext}_{c}^{t}\left(X ; F, K_{\dot{X}}\right)$ is a Hausdorff space if and only if, for every $K \subset X, K$ compact, there exists a compact subset $K^{\prime}$ containing $K$ such that every element in $\operatorname{Ext}_{K}^{j}\left(X ; F, K_{\dot{X}}\right)$, whose image in $\operatorname{Ext}_{c}^{j}\left(X ; F, K_{\dot{X}}\right)$ is zero, already has image zero in $\operatorname{Ext}_{K^{\prime}}^{j}\left(X ; F, K_{X}\right)$.

Finally let us denote by $\operatorname{Ext}\left(X ; F, K_{\dot{X}}\right), F \in \operatorname{Coh}(X)$, the sheaf whose fiber at the point $x \in X$ is $\operatorname{Ext}_{x}^{\circ}\left(X ; F, K_{\dot{X}}\right)$.

(0.6) We shall say that $X$ is a cohomologically $q$-complete ( $q$-convex) space if $H^{r}(X, F)=0\left(\operatorname{dim} H^{r}(X, F)<+\infty\right)$ for every $F \in \operatorname{Coh}(X)$ and for every $r, r \geqslant q$. Then $X$ a Stein space is equivalent to $X$ cohomologically 1-complete. We have the following deep properties:

(a) (Siu [11]). $\left({ }^{1}\right)$ If $X$ has no (only a finite number of) compact irreducible component then $H^{n}(X, F)=0\left(\operatorname{dim} H^{n}(X, F)<+\infty\right)$ for every $F \in \operatorname{Coh}(X)$.

(b) (Reiffen [8]). Every analytic space is cohomologically $(n+1)$-complete. Combining (a) and (b), we get the following statement:

THEOREM 0. If $X$ has no (only a finite number of) compact irreducible component, then $X$ is cohomologically n-complete (n-convex).

(1) In Siu's proof $X$ is not necessarily reduced. 
(0.7) Now let us introduce the main definition, whose discussion is the object of the present paper: Let $q, q \geqslant 1$, a fixed integer; we say that $(X, Y)$ is a q-Runge pair of complex analytic spaces if the restriction homomorphism

$$
\rho_{Y}^{X}: H^{q-1}\left(X, \Omega^{p}\right) \rightarrow H^{q-1}\left(Y, \Omega^{p}\right)
$$

has dense image for every $p=0,1, \cdots, n$.

1. On the main definition.

(1.1) If $X$ is a manifold, the following proposition shows that the main definition agrees with the definition of a $q$-Runge pair of complex analytic manifolds first given in [12].

PROPOSITION. The following statements are equivalent:

(i) The restriction homomorphism $r_{Y}^{X}: \Gamma\left(X, \operatorname{ker} d_{p, q-1}^{\prime \prime}\right) \rightarrow \Gamma\left(Y, \operatorname{ker} d_{p, q-1}^{\prime \prime}\right)$ has dense image for every $p=0,1, \cdots, n$ (i.e. $(X, Y)$ is a q-Runge pair of manifolds according to [12]).

(ii) The restriction homomorphism $\rho_{Y}^{X}: H^{q-1}\left(X, \Omega^{p}\right) \rightarrow H^{q-1}\left(Y, \Omega^{p}\right)$ has dense image for every $p=0,1, \cdots, n$ (i.e. $(X, Y)$ is a q-Runge pair of spaces according to the main definition).

(iii) The restriction homomorphism $\stackrel{r}{\rho}_{Y}^{X}: \check{H}^{q-1}\left(X, \Omega^{p}\right) \rightarrow h^{q-1}\left(Y, \Omega^{p}\right)$ has dense image for every $p=0,1, \cdots, n$.

PRoof. If $q=1, \Gamma\left(X[Y], \operatorname{ker} d_{p, 0}^{\prime \prime}\right)=H^{0}\left(X[Y], \Omega^{p}\right)=h^{0}\left(X[Y], \Omega^{p}\right)$, and there is nothing to prove. Suppose then $q>1$ and let us consider the following diagrams:

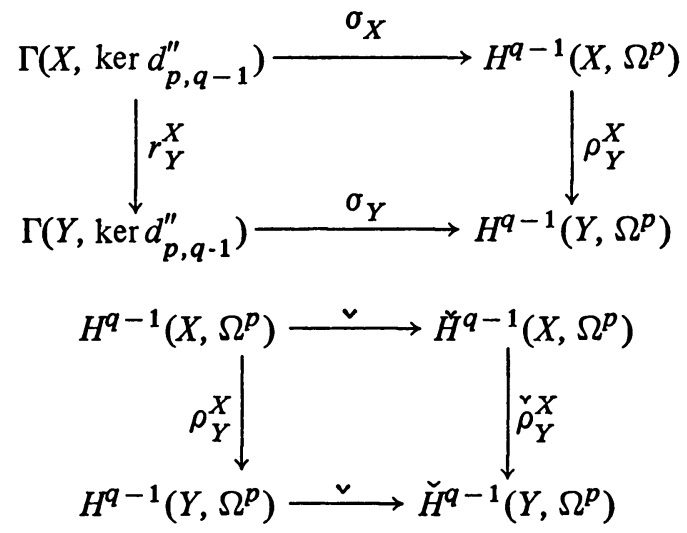

If (i) holds, $\sigma_{X}, \sigma_{Y}$ surjective implies that $\rho_{Y}^{X}$ has dense image, i.e.(ii) holds. If (ii) holds, the separation homomorphism being surjective, we have that $\stackrel{r}{\rho}_{Y}^{X}$ has dense image, i.e. (iii) holds.

It remains to prove that (iii) implies (i); this follows from:

(1.2) LeMMA. Let $E_{i} \stackrel{u_{i}}{\longrightarrow} F_{i} \stackrel{v_{i}}{\longrightarrow} G_{i}, i=1,2$, be two sequences of 
Fréchet spaces and linear continuous maps with $v_{i} \circ u_{i}=0, i=1,2$, in the following situation:

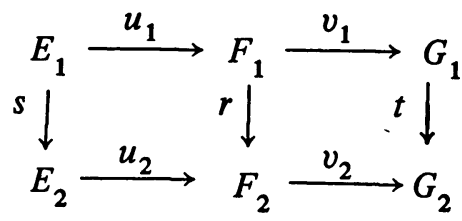

where $r, s, t$ are restriction homomorphisms with dense image commuting with $u_{i}$ and $v_{i}, i=1,2$. Then if the induced restriction homomorphism $\stackrel{\rho}{\circ}$ : $\left(\operatorname{ker} v_{1} / \overline{\operatorname{Im} u_{1}}\right) \rightarrow\left(\operatorname{ker} v_{2} / \overline{\operatorname{Im} u_{2}}\right)$ has dense image, $r\left(\operatorname{ker} v_{1}\right)$ is dense in $\operatorname{ker} v_{2}$.

Proof. Let us call $\pi_{i}, i=1,2$, the natural maps $\pi_{i}$ : $\operatorname{ker} v_{1} \rightarrow \operatorname{ker} v_{i}{\overline{\operatorname{Im}} u_{i}}_{\text {. }}$. As $\not \delta$ has dense image, for every $f^{(2)} \in$ ker $v_{2}$, there exists a sequence $\left\{f_{n}^{(1)}\right\}_{n \in \mathrm{N}}, f_{n}^{(1)} \in \operatorname{ker} v_{1}$ for every $n \in \mathrm{N}$, such that $\pi_{2}\left(r\left(f_{n}^{(1)}\right)-f^{(2)}\right) \rightarrow 0$. The homomorphisms $\pi_{i}, i=1,2$, are surjective, hence topological, then there exists a sequence $\left\{f_{n}^{(2)}\right\}_{n \in \mathrm{N}}, f_{n}^{(2)} \in \operatorname{ker} v_{2}$ for every $n$, such that $f_{n}^{(2)} \rightarrow 0$ and $\pi_{2}\left(r\left(f_{n}^{(1)}\right)-f^{(2)}\right)=\pi_{2}\left(f_{n}^{(2)}\right)$ for every $n \in \mathrm{N}$, i.e. $\pi_{2}\left(r\left(f_{n}^{(1)}\right)-f^{(2)}-f_{n}^{(2)}\right)$ $=0$, therefore $\left(r\left(f_{n}^{(1)}\right)-f^{(2)}-f_{n}^{(2)}\right) \in \operatorname{Im} u_{2}$ for every $n \in N$.

Then there exists a sequence $\left\{e_{n}^{(2)}\right\}_{n \in \mathrm{N}}, e_{n}^{(2)} \in E_{2}, \forall n$, such that $\left(u_{2}\left(e_{n}^{(2)}\right)-\left(r\left(f_{n}^{(1)}\right)-f^{(2)}-f_{n}^{(2)}\right)\right) \rightarrow 0$, and, by the density of $\operatorname{Im}(s)$, a sequence $\left\{e_{n}^{(1)}\right\}_{n \in \mathrm{N}}, e_{n}^{(1)} \in E_{1}$ for every $n$, such that

$$
\left(u_{2}\left(s\left(e_{n}^{(1)}\right)\right)-\left(r\left(f_{n}^{(1)}\right)-f^{(2)}-f_{n}^{(2)}\right)\right) \rightarrow 0 .
$$

But $u_{2}\left(s\left(e_{n}^{(1)}\right)\right)=r\left(u_{1}\left(e_{n}^{(1)}\right)\right)$ and we have

$$
\begin{gathered}
\lim _{n}\left(r\left(f_{n}^{(1)}\right)-f^{(2)}-f_{n}^{(2)}-r\left(u_{1}\left(e_{n}^{(1)}\right)\right)\right)=0 \text { i.e. } \\
\lim _{n} r\left(f_{n}^{(1)}-u_{1}\left(e_{n}^{(1)}\right)\right)-f_{n}^{(2)}=f^{(2)}
\end{gathered}
$$

but $\lim _{n} f_{n}^{(2)}=0$, then $\lim r\left(f_{n}^{(1)}-u_{1}\left(e_{n}^{(1)}\right)\right)=f^{(2)}$. Q.E.D.

Making use of Dolbeault's resolution of $\Omega^{p}$, the hypotheses of the lemma are fullfilled and so we get the proposition.

(1.4) The main definition also agrees with the usual definition of a Runge pair of Stein spaces (i.e. the restriction homomorphism $r: \Gamma(X, 0) \rightarrow \Gamma(Y, 0)$ has dense image, f.i. see [2]). Indeed we have

Proposition. $(X, Y)$ is a 1-Runge pair of cohomologically 1-complete spaces if and only if $(X, Y)$ is a Runge pair of Stein spaces.

If $(X, Y)$ is a 1 -Runge pair of cohomologically 1 -complete spaces, trivially $(X, Y)$ is a Runge pair of Stein spaces.

Conversely, let us consider the following diagram: 


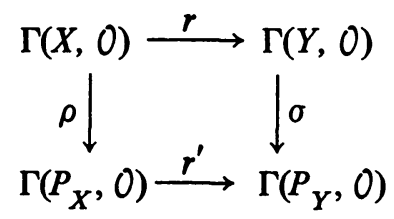

where the arrows are restriction homomorphisms and $P_{X} \subset X, P_{Y} \subset Y$, are analytical polyhedra, with $P_{X} \supset P_{Y}$.

As $r, \rho, \sigma$ have dense image, by easy computation $r^{\prime}$ has dense image.

Let $F \in \operatorname{Coh}(X)$; we have (on $P_{X}$ and $P_{Y}$ ) the sheaf epimorphisms:

$$
0^{d} X \rightarrow F \rightarrow 0, \quad O^{d} Y \rightarrow F \rightarrow 0 .
$$

Since $P_{X} \cap P_{Y}=P_{Y}$, we can take $d=d_{X}=d_{Y}$. By Theorem B the horizontal arrows of the following diagram are surjective:

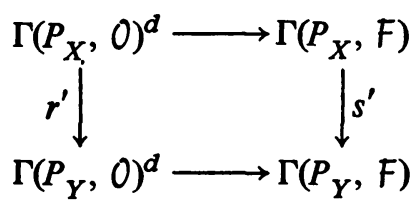

$r^{\prime}$ having dense image, then $s^{\prime}$ has dense image; but $\rho^{\prime}: \Gamma(X, F) \rightarrow \Gamma\left(P_{X}, F\right)$ has dense image (cover $X$ by an increasing sequence of analytical polyedra with same center then $\left.P_{X}\right)$, then $s=s^{\prime} \circ \rho^{\prime}: \Gamma(X, F) \rightarrow \Gamma\left(P_{Y}, F\right)$ has dense image.

Since for every compact subset $K, K \subset Y$, there exists an analytic polyhedron $P_{Y}$ such that $K \subset P_{Y} \subset \subset Y$; we can conclude that the restriction $\Gamma(X, F) \rightarrow \Gamma(Y, F)$ has dense image. Taking $F=\Omega^{p}$ for every $p=0,1$, $\cdots, n$ we get the proposition.

\section{Behnke-Stein "Rungescherapproximationssatz" for spaces. Suppose} that $X$ has no compact irreducible component, then the main result of this section is the following:

THEOREM (2.1). If $X-Y$ has no compact irreducible component, then $(X, Y)$ is an $n$-Runge pair.

Proof. (I) The following statements are equivalent:

(a) $(X, Y)$ is a q-Runge pair of analytic spaces.

(b) The restriction homomorphism $\stackrel{r}{\rho_{q}}: \check{H}^{q-1}\left(X, \Omega^{p}\right) \rightarrow \stackrel{\check{H}^{q-1}}{ }\left(Y, \Omega^{p}\right)$ has dense image for every $p=0,1, \cdots, n$.

Use as proof the one of $\S \S(1.1),(1.2)$, replacing Dolbeault's resolution by some acyclic Fréchet resolution of $\Omega^{p}$ and taking into account Leray's theorem for Hausdorff cohomology (see [3]).

(II) $\check{\rho}_{n}$ has dense image if and only if $\operatorname{Ext}_{c}^{-n}\left(X-Y ; \Omega^{p}, K_{\dot{X}}\right)=0 . \check{\rho}_{n}$ has dense image if and only if (by théorème de dualité $(0.4)$ ) 


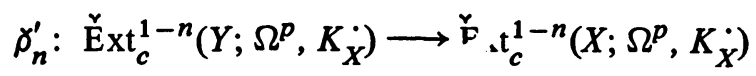

is injective.

But, by Theorem 0 and théorème de dualité, $\mathrm{Ext}_{c}^{1-n}\left(X ; \Omega^{p}, K_{\dot{X}}\right)=$ $\operatorname{Ext}_{c}^{1-n}\left(X ; \Omega^{p}, K_{\dot{X}}\right)$ and $\rho_{n}^{\prime}=\rho_{n}^{\prime}$, and the same holds for $Y$, since $Y$ also has no $n$-dimensional compact branch.

Now let us consider the $\mathrm{Ext}_{c}{ }_{c}$ exact sequence:

$$
\begin{aligned}
\cdots & \longrightarrow \operatorname{Ext}_{c}^{-n}\left(Y ; \Omega^{p}, K_{X}\right) \longrightarrow \operatorname{Ext}_{c}^{-n}\left(X ; \Omega^{p}, K_{X}\right) \longrightarrow \\
& \longrightarrow \operatorname{Ext}_{c}^{-n}\left(X-Y ; \Omega^{p}, K_{X}^{*}\right) \longrightarrow \operatorname{Ext}_{c}^{1-n}\left(Y ; \Omega^{p}, K_{X}\right) \longrightarrow \\
& \longrightarrow \operatorname{Ext}_{c}^{1-n}\left(X ; \Omega^{p}, K_{X}\right) \longrightarrow \cdots
\end{aligned}
$$

from which we get that $\rho_{n}^{\prime}$ is injective if and only if $\operatorname{Ext}_{c}^{-n}\left(X-Y ; \Omega^{p}, K_{X}\right)$ $=0$, since by Theorem 0 and dualizing we have that

$$
\operatorname{Ext}_{c}^{-n}\left(X ; \Omega^{p}, K_{X}^{*}\right)=\operatorname{Ext}_{c}^{-n}\left(Y ; \Omega^{p}, K_{X}^{\dot{*}}\right)=0 .
$$

(III) If $X-Y$ has no compact irreducible component, then $\operatorname{Ext}_{c}^{-n}\left(X-Y ; \Omega^{p}, K_{\dot{X}}\right)=0$. The spectral sequence of the composed functor $\Gamma_{c} \cdot$ Hom gives

(*) $\quad \operatorname{Ext}_{c}^{-n}\left(X-Y ; \Omega^{p}, K_{X}\right)=\Gamma_{c}\left(X-Y, \operatorname{Ext}^{-n}\left(X ; \Omega^{p}, K_{X}\right)\right)$.

From [7, Lemma 1, Appendix] we know that the sheaf $\operatorname{Ext}^{-n}\left(X ; F, K_{\dot{X}}\right)$, $F \in \operatorname{Coh}(X)$, is torsion free.

Now the support of a section of a torsion-free sheaf is the closure of the union of some irreducible component of $X-Y$, then the support cannot be compact, hence $\Gamma_{c}\left(X-Y, E x t^{-n}\left(X ; \Omega^{p}, K_{X}\right)\right)=0$, and the proof of Theorem (2.1) is complete.

(2.2) The converse of part (III) and then of Theorem (2.1) can be proved f.i. under the additional hypothesis $\Gamma\left(X, \operatorname{Ext} t^{-n}\left(X ; \Omega^{p}, K_{X}\right)\right) \neq 0$. Indeed suppose that $X-Y$ has a compact irreducible component, say $X_{1}$. We have $X-Y=X_{1} \cup X_{2}$ and $X_{1} \cap X_{2}=\varnothing$. Then take the zero section of $\operatorname{Ext}^{-n}\left(X ; \Omega^{p}, K_{X}\right)$ on $X_{2}$ and the restriction to $X_{1}$ of some nonzero element in $\Gamma\left(X, \operatorname{Ext}^{-n}\left(X ; \Omega^{p}, K_{X}\right)\right)$; this is absurd, since from $(*)$, we know that $\Gamma_{c}\left(X-Y ; \operatorname{Ext}^{-n}\left(X ; \Omega^{p}, K_{X}\right)\right)=0$.

(2.3) REMARK. If one does not like the assumption " $X$ has no compact irreducible component," he can replace it by $H^{n}\left(X, \Omega^{p}\right)=H^{n}\left(Y, \Omega^{p}\right)=0$, for $p=0,1, \cdots, n$.

(2.4) For $X$ a manifold, Theorems (2.1) and (2.2) are proved in [10]. The topological condition in this case can be given in the following form: $X-Y$ has no compact connected components.

Indeed let us denote by $\operatorname{Reg}(M)$ the set of regular points of a space $M$. If $C$ is connected component of $\operatorname{Reg}(M)$, an irreducible branch of $M$ is the 
closure $\bar{C}$ of $C$. If $M$ is a manifold, $\operatorname{Reg}(M)=M$; therefore if $X-Y$ has no compact irreducible branch and since $\operatorname{Reg}(X-Y)=X-Y, X-Y$ has no compact connected components. This condition is sometimes replaced in the literature by the equivalent condition: $X-Y$ has no nonempty, relatively open, compact subsets. For $n=1$, in view of (1.4), we get the classical Behnke-Stein "Rungescherapproximationssatz" for Riemann surfaces.

(2.5) For $q \neq n$, Theorem (2.1) is not true. We have some well-known counterexamples; take for instance 0 . Forster's one (Eine Bemerkung über Rungesche Paure, Ark. Mat. 14 (1963), 334-336): set $X=\mathrm{C}^{2}, Y=\left\{\left(z_{1}, z_{2}\right) \in \mathrm{C}^{2}\right.$ s.t. $\left.1-\epsilon<\left|z_{1}\right|<1+\epsilon,\left|z_{2}\right|<\epsilon\right\}$ with $0<\epsilon \leqslant 5 \cdot 10^{-3}$.

We have $H^{r}\left(X, \Omega^{p}\right)=H^{r}\left(Y, \Omega^{p}\right)=0$ for $p=0,1,2$ and $r=1,2 ; X-Y$ is an unbounded connected subset of $\mathbf{C}^{2}$, hence $(X, Y)$ is a 2-Runge pair, but $(X, Y)$ is not a $1-$ Runge pair.

3. A topological property of $q$-Runge pairs of analytic spaces.

(3.1) The following statement generalizes a result obtained in [12] for manifolds:

THEOREM. If $(X, Y)$ is a q-Runge pair of cohomologically q-complete spaces, then $H_{i}(X \bmod Y, \mathbf{C})=0$ for $i \geqslant n+q$.

Proof. We need the following results:

( $\alpha$ ) (Ferrari [4]). If $X$ is a cohomologically $q$-complete space, then $H_{i}(X, \mathrm{C})=0$ for $i \geqslant n+q$.

( $\beta$ ) (Le Potier [5]). There exists a canonical morphism $\vartheta$

$$
\vartheta: H^{q-1}\left(X, \Omega^{n}\right) \rightarrow H^{n+q-1}(X, \mathrm{C})
$$

such that if $X$ is cohomologically $q$-complete, $\vartheta$ is surjective.

From the exact homology sequence we get

$$
H_{i}(Y, \mathrm{C}) \rightarrow H_{i}(X, \mathrm{C}) \rightarrow H_{i}(X \bmod Y, \mathrm{C}) \rightarrow H_{i-1}(Y, \mathrm{C}) .
$$

For $i>n+q$, by $(\alpha)$, we have $H_{i}(X \bmod Y, \mathrm{C})=0$.

Now suppose $i=n+q$. We have $H_{n+q}(X \bmod Y, \mathrm{C})=0$ if and only if $\rho^{*}: H_{n+q-1}(Y, \mathrm{C}) \rightarrow H_{n+q-1}(X, \mathrm{C})$ is injective. But $\rho^{*}$ is injective if and only if $\rho_{Y}^{X}: H^{n+q-1}(X, \mathbf{C}) \rightarrow H^{n+q-1}(Y, \mathbf{C})$ has dense image.

Let us consider the following diagram:

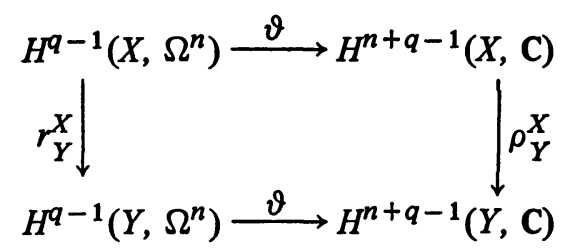

$r_{Y}^{X}$ has dense image by assumption and $\vartheta$ being surjective for $(\beta)$, we have $\rho_{Y}^{X}$ has dense image and the theorem is proved. 
4. An application. In this section we are going to extend to a class of analytic spaces two results proved in [9] for manifolds.

THEOREM (4.1). Let $X$ be an analytic space of complex dimension $n, X=$ $\bigcup_{i \in \mathrm{N}} X_{i}, X_{i}$ open in $X$ for every $i=0,1, \cdots, q \geqslant 1$ a fixed integer, such that

(i) $X_{i} \subset \subset X_{i+1}$ for every $i \in \mathbf{N}$,

(ii) $\left(X_{i+1}, X_{i}\right)$ is a q-Runge pair for every $i \in \mathbf{N}$.

Then if $H^{q}\left(X_{i}, \Omega^{p}\right)=0$ for every $i \in \mathbf{N}, p \geqslant 0$, we have also $H^{q}\left(X, \Omega^{p}\right)=0$.

We know that

(*) (Cassa [3]). $\check{H}^{q}(X, F)$ is always trivial for every $F \in \operatorname{Coh}(X)$. Therefore Theorem (4.1) will be proved iff we prove that $H^{q}\left(X, \Omega^{p}\right)$ is a Hausdorff space.

Now, if $\left(X_{i+1}, X_{i}\right)$ is a $q$-Runge pair for every $i$, by [1, Lemma for Proposition 20] $\left(X, X_{i}\right)$ is a $q$-Runge pair for every $i \in \mathrm{N}$. By (2.1), part (I), we also know that $\stackrel{r}{\rho}$ has dense image and, dualizing, $\stackrel{\Upsilon}{\rho}$ has dense image if and only if

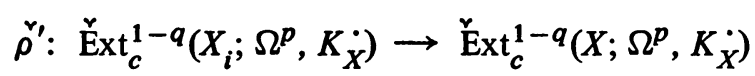

is injective for every $i \in \mathrm{N}$. But $\breve{E x t}_{c}^{1-q}\left(X_{i} ; \Omega^{p}, K_{\dot{X}}\right)=\operatorname{Ext}_{c}^{1-q}\left(X_{i} ; \Omega^{p}, K_{\dot{X}}\right)$ by duality; then from the following diagram:

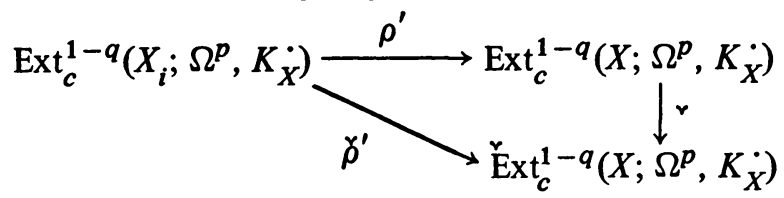

we get $\rho^{\prime}$ injective for every $i \in \mathbf{N}$.

Let $K, K \subset X$, a compact subset; there exists $\bar{i} \in \mathbf{N}$, s.t. $K \subset X_{\bar{i}} \subset \bar{X}_{\bar{i}}$ $\subset X$. Let us consider the following diagram and apply criterion (0.5):

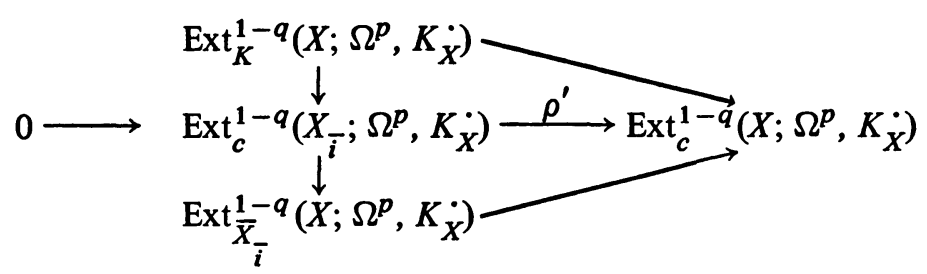

It is clear that an element in $\operatorname{Ext}_{K}^{1-q}\left(X ; \Omega^{p} ; K_{X}^{\cdot}\right)$ which gives zero in $\operatorname{Ext}_{c}^{1-q}\left(X ; \Omega^{p}, K_{\dot{X}}\right)$, gives zero in $\operatorname{Ext}_{c}^{1-q}\left(X_{\bar{i}} ; \Omega^{p}, K_{X}\right)$ then is zero in $\left.\operatorname{Ext}_{\bar{X}_{i}} \frac{1-q}{\left(X ; \Omega^{p}\right.}, K_{X}\right)$ and $(0.5)$ is verified.

By théorème de dualité, (4.1) is then proved.

Theorem (4.2). Let $X$ be an analytic space open in a Stein space $M, X=$ $\cup_{i \in \mathrm{N}} X_{i}, X_{i}$ open in $X$ for every $i \in \mathrm{N}, X_{i}$ cohomologically q-complete for $q \geqslant 1$ fixed, $i=0,1, \cdots$, such that 
(i) $X_{i} \subset \subset X_{i+1}$ for every $i \in \mathrm{N}$.

(ii) $\left(X_{i+1}, X_{i}\right)$ is a q-Runge pair for every $i \in \mathrm{N}$.

Then $\forall F \in \operatorname{Coh}(M)$, we have $H^{r}(X, F)=0, r \geqslant q$.

Proof. For $r>q$ we have that $H^{r}(X, F)=0$ for every $F \in \operatorname{Coh}(X)$ by means of standard arguments which make use of the canonical flabby resolution of $F$. Consider now the case $r=q$. Since $M$ is Stein, by Cartan's Theorem A we have that, for every $F \in \operatorname{Coh}(M)$ restricted to $\operatorname{Coh}(X)$ and for every $x \in X$, the $O_{X, x}$-module $F_{x}$ is generated by the image of $\Gamma(M, F)$ in $F_{x}$ under the canonical map. Let $x \in X_{i+1}$; there exists a finite set of elements in $\Gamma\left(X_{i+1}, F\right)$ whose image in $F_{x}$ generates $F_{x}$; since $F$ is coherent these elements generate $F_{y}$ for every $y$ in an open neighborhood of $x$ and since $\bar{X}_{i}$ is compact, the image of a finite number of elements in $\Gamma\left(X_{i+1}, F\right)$ generates $F_{y}$ for every $y$ in an open neighborhood of $\bar{X}_{i}$, so we get the exact sequence of sheaves on $X_{i}$ :

$$
0 \longrightarrow \mathrm{R} \longrightarrow \mathrm{O}^{p} \longrightarrow \mathrm{F} / X_{i+1} \rightarrow 0 .
$$

Since $X_{i}$ is supposed cohomologically $q$-complete for every $i \in \mathbf{N}$, from the exact cohomology sequence we get that the homomorphism: $H^{q-1}\left(X_{i}, O^{p}\right) \rightarrow$ $H^{q-1}\left(X_{i}, F\right)$ is surjective for every $i \in \mathbf{N}$. Then the restriction homomorphism $\sigma: H^{q-1}\left(X_{i+1}, F\right) \rightarrow H^{q-1}\left(X_{i}, F\right)$ has dense image for every $i \in \mathbf{N}$, and ò: $\check{H}^{q-1}\left(X_{i+1}, F\right) \rightarrow \check{H}^{q-1}\left(X_{i}, F\right)$ has dense image for every $i \in \mathbf{N}$. We are doing now with Fréchet spaces, then by $[1$, loc. cit] we have also that the homomorphism $\check{H}^{q-1}(X, F) \rightarrow \check{H}^{q-1}\left(X_{i}, F\right)$ has dense image for every $i \in \mathbf{N}$. By (4.1) (*), it is sufficient to apply criterion (0.5) for every $F \in \operatorname{Coh}(M)$; and if we proceed as in (4.1) with $F$ instead of $\Omega^{p}$ we get the theorem.

(4.3) In [9] an example shows that if we do not suppose $F \in \operatorname{Coh}(M)$ there exists a coherent sheaf $G$ on $X$ with $H^{q}\left(X_{i}, G\right)=P$ but $H^{q}(X, G) \neq 0$ and $\left(X, X_{i+1}\right)$ a $q$-Runge pair.

\section{BIBLIOGRAPHY}

1. A. Andreotti and H. Grauert, Théorèmes de finitude pour la cohomologie des espaces complexes, Bull. Soc. Math. France 90 (1962), 193-259. MR 27 \#343.

2. A. Andreotti and R. Narasimhan, A topological property of Runge pairs, Ann. of Math. (2) 76 (1962), 499-509. MR 25 \#4128.

3. A. Cassa, Coomologia separata sulle varietà analitiche complesse, Ann. Scuola Norm. Sup. Pisa (3) 25 (1971), 291-323.

4. A. Ferrari, Cohomology and holomorphic differential forms on complex analytic spaces, Ann. Scuola Norm. Sup. Pisa (3) 24 (1970), 65-77. MR 43 \#570.

5. J. Le Potier, Une propriété topologique des espaces q-convexes, Bull. Soc. Math. France 98 (1970), 319-328. MR 43 \#7664.

6. J. P. Ramis and G. Ruget, Complexe dualisant et théorèmes de dualité en géométrie analytique complexe, Inst. Hautes Etudes Sci. Publ. Math. No. 38 (1971), 77-91. MR 43 \#5060. 
7. J. P. Ramis, G. Ruget and J. L. Verdier, Dualité relative en géométrie analytique complexe, Invent. Math. 13 (1971), 261-283. MR 46 \#7553.

8. H.-J. Reiffen, Riemannsche Hebbarkeitssätze für Cohomologieklassen mit kompakten Trager, Math. Ann. 164 (1966), 272-279. MR 33 \#5942.

9. A. Silva, Successioni crescenti di q-coppie di Runge di varietà analitiche complesse, Ann. Scuola Norm. Sup. Pisa (3) 27 (1973), 431-440.

10.

$\longrightarrow$, A n-Runge approximation theorem, Boll. Un. Mat. Ital. 8 (1973), 286-289.

11. Y. T. Siu, Analytic sheaf cohomology groups of dimension $n$ of $n$-dimensional complex spaces, Trans. Amer. Math. Soc. 143 (1969), 77-94. MR 40 \#5902.

12. G. Sorani, Homologie des q-paires de Runge, Ann. Scuola Norm. Sup. Pisa (3) 17 (1963), 319-332. MR 29 \#2432.

ISTITUTO DI MATEMATICA, UNIVERSITÀ DI GENOVA, GENOVA, ITALY 